\title{
FOLH1/GCPII is elevated in IBD patients, and its inhibition ameliorates murine IBD abnormalities
}

\author{
Rana Rais,,$^{1,2}$ Weiwei Jiang, ${ }^{3,4}$ Huihong Zhai, ${ }^{3}$ Krystyna M. Wozniak, ${ }^{2}$ Marigo Stathis, ${ }^{2}$ \\ Kristen R. Hollinger, ${ }^{1,5}$ Ajit G. Thomas, ${ }^{2}$ Camilo Rojas, ${ }^{2,6}$ James J. Vornov, ${ }^{7}$ Michael Marohn, ${ }^{8}$ \\ Xuhang Li, ${ }^{3}$ and Barbara S. Slusher ${ }^{1,2,3,5}$ \\ 'Department of Neurology, ${ }^{2}$ Johns Hopkins Drug Discovery, and ${ }^{3}$ Department of Medicine, Johns Hopkins University School \\ of Medicine, Baltimore, Maryland, USA. ${ }^{4}$ Division of Gastroenterology, Union Hospital, Tongji Medical College, Huazhong \\ University of Science and Technology, Wuhan, China. ${ }^{5}$ Department of Psychiatry and ${ }^{6}$ Department of Molecular and \\ Comparative Pathobiology, Johns Hopkins University School of Medicine, Baltimore, Maryland, USA. ${ }^{7}$ Medpace, Cincinnati, \\ Ohio, USA. ${ }^{8}$ Department of Surgery, Johns Hopkins University School of Medicine, Baltimore, Maryland, USA.
}

Recent gene-profiling analyses showed significant upregulation of the folate hydrolase (FOLH1) gene in the affected intestinal mucosa of patients with inflammatory bowel disease (IBD). The FOLH1 gene encodes a type II transmembrane glycoprotein termed glutamate carboxypeptidase II (GCPII). To establish that the previously reported increased gene expression was functional, we quantified the glutamate carboxypeptidase enzymatic activity in 31 surgical specimens and report a robust 2.8- to 41-fold increase in enzymatic activity in the affected intestinal mucosa of IBD patients compared with an uninvolved area in the same patients or intestinal mucosa from healthy controls. Using a human-to-mouse approach, we next showed a similar enzymatic increase in two well-validated IBD murine models and evaluated the therapeutic effect of the potent FOLH1/ CCPII inhibitor 2-phosphonomethyl pentanedioic acid (2-PMPA) (IC $\left.C_{50}=300 \mathrm{pM}\right)$. In the dextran sodium sulfate (DSS) colitis model, 2-PMPA inhibited the GCPII activity in the colonic mucosa by over $90 \%$ and substantially reduced the disease activity. The significance of the target was confirmed in $\mathrm{FOLH1}^{-/-}$mice who exhibited resistance to DSS treatment. In the murine $\mathrm{IL}^{-10^{-/-}}$model of spontaneous colitis, daily 2-PMPA treatment also significantly reduced both macroscopic and microscopic disease severity. These results provide the first evidence of FOLH1/CCPII enzymatic inhibition as a therapeutic option for IBD.

Authorship note: R. Rais, W. Jiang, and $H$. Zhai contributed equally to this work.

Conflict of interest: B.S. Slusher, R. Rais, and X. Li filed a patent application covering the use of FOLH1/GCPII inhibitors as IBD therapeutics (PCT/ US2015/044025).

Submitted: May 18, 2016 Accepted: July 1, 2016 Published: August 4, 2016

Reference information: JCI Insight. 2016;1(12):e88634 doi:10.1172/jci.insight.88634.

\section{Introduction}

Inflammatory bowel disease (IBD) is an idiopathic, chronic, and frequently disabling inflammatory disorder of the intestine that has two subtypes: Crohn's disease (CD) and ulcerative colitis (UC), each accounting for approximately $50 \%$ of IBD patients (1-3). In the United States alone, there are 1.4 million diagnosed IBD patients. It is clear that IBD is a complex multifactorial disease with both genetic and environmental contributions (1-4), yet the precise etiology of the mucosal dysregulation remains elusive (4). Despite the therapeutic options available for the management of IBD, approximately one-third of IBD patients do not respond to any given therapy, and there is no cure (5). This emphasizes the significance of exploring and identifying novel therapies for patients with IBD.

A novel therapeutic approach was recently suggested by the finding that the expression of the FOLH1 gene is dramatically increased in IBD (6-8). Using an unbiased statistical analysis of genome-wide expression data from biopsy samples, the FOLH1 gene was identified as a "hub" gene, with significant correlations to over a dozen known IBD gene biomarkers. Immunohistochemical staining confirmed the elevated expression of the FOLH1 protein in the villous epithelium of samples from patients when compared with non-IBD controls. These findings suggest that FOLH1 might serve as a biomarker for disease and could serve as a possible therapeutic target.

FOLH1 encodes a transmembrane glycoprotein that acts as a glutamate carboxypeptidase. In the intestines, the enzyme is called folate hydrolase and is found on brush border membranes where it is involved in 
A

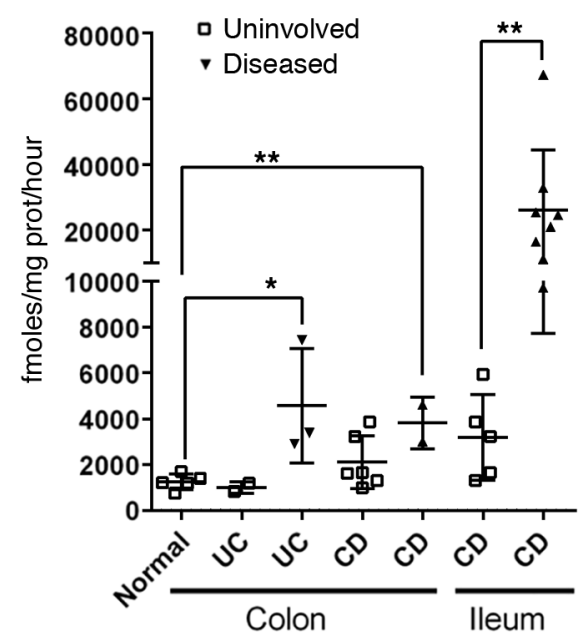

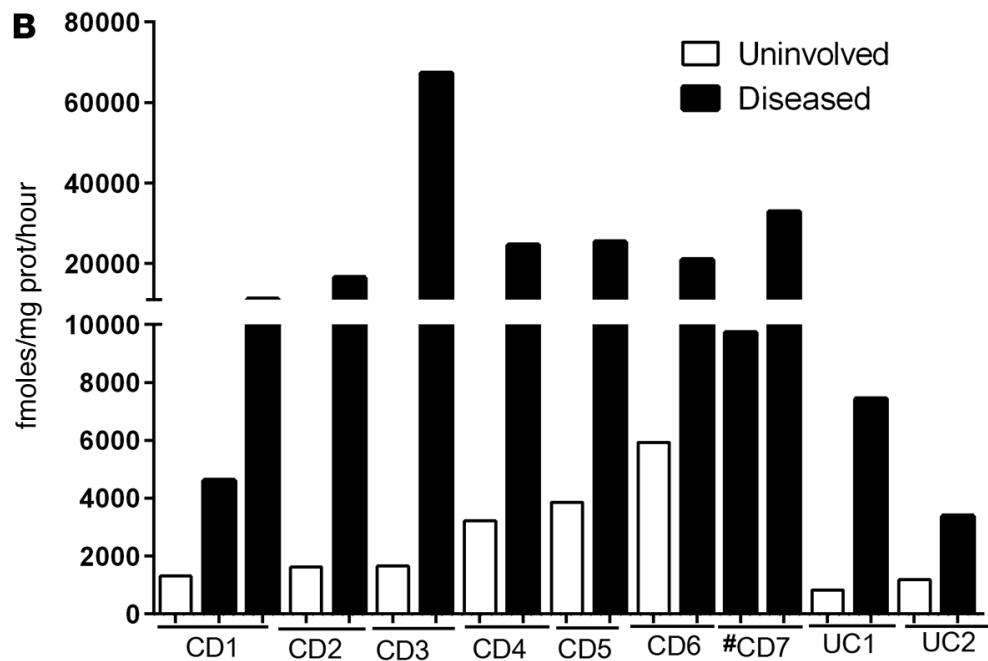

Figure 1. Elevation of FOLH1/glutamate carboxypeptidase II activity in the diseased intestinal mucosa of patients with IBD. FOLH1/glutamate carboxypeptidase II (FOLH1/GCPII) enzymatic activity was measured in involved (inflamed with active disease) and uninvolved (macroscopically normal) mucosal specimens from IBD patients or from non-IBD controls. (A) Significant enhancement of GCPII activity was observed in diseased mucosal specimens taken from Crohn's disease (CD) and ulcerative colitis (UC) patients when compared with specimens from normal healthy controls in both colon and ileum. Data are shown as mean \pm SEM ( $n=31$ samples) $\left({ }^{*} P<0.05,{ }^{* *} P<0.01\right.$, 2-tailed $t$ test). (B) Within the same IBD patient, a robust increase in enzymatic activity was observed in the diseased intestinal mucosa specimens when compared with that in an uninvolved region from the same patient. Data are presented as individual specimens $(n=19)$. The Arabic numbers refer to different patients. The pound sign indicates that an uninvolved normal region from this patient was not available.

the sequential cleavage of terminal $\gamma$-linked glutamate residues from dietary polyglutamyl folates to enable the absorption of folate. In addition to the gut, the enzyme is also highly expressed in prostate cancer and in the neovasculature of solid tumors, in which it is termed prostate-specific membrane antigen (PSMA) and serves as a cancer biomarker (9-11). In the brain and peripheral nervous system, in which it cleaves the abundant dipeptide neurotransmitter N-acetylaspartylglutamate (NAAG) to liberate glutamate $(12,13)$, the enzyme is referred to as glutamate carboxypeptidase II (GCPII). Multiple classes of potent and selective small-molecule GCPII inhibitors have been synthesized (14-18) and shown to have profound therapeutic effects in a variety of preclinical models of neurological disorders wherein excess glutamate is implicated (18-22).

Herein, we report that the previously described increased FOLH1 gene expression results in a large and significant increase of glutamate carboxypeptidase activity selectively in the affected intestinal mucosa of patients with both active CD and UC. In addition, a similar degree of increase in enzymatic activity was detected in preclinical models of colitis. Given the correlation in human and IBD preclinical models, we next evaluated the effect of pharmacological inhibition of FOLH1/GCPII using the potent and selective inhibitor 2-PMPA. We demonstrate that systemic administration of 2-PMPA blocks the colonic glutamate carboxypeptidase activity and ameliorates IBD symptoms in two widely used murine preclinical models. Last, we validated FOLH1/GCPII as a therapeutic target by showing that $\mathrm{FOLH1}^{-1-}$ mice are significantly protected against experimental colitis.

\section{Results}

Increased FOLH1/GCPII enzymatic activity in IBD patients. FOLH1/GCPII enzymatic activity was evaluated in 31 surgical specimens from 20 subjects with either CD or UC or from normal controls. When comparing to normal colon from healthy volunteers, the enzymatic activity was robustly increased in diseased colon from both UC $(3.6 \pm 1.2$-fold; $P<0.05)$ and CD (3.04 \pm 0.73 -fold; $P<0.01)$ subjects. An even larger increase in enzymatic activity was observed in diseased ileum tissue $(8.6 \pm 3.5$-fold; $P<0.01)$ (Figure 1A). When comparing FOLH1/GCPII activity in diseased versus uninvolved intestinal mucosa specimens from the same patients, we observed a robust 2.8- to 41-fold increase associated with disease (Figure 1B).

Increased FOLH1 / GCPII enzymatic activity in murine models of colitis. FOLH1/GCPII enzymatic activity was evaluated in the colon and ileum of dextran sodium sulfate-treated (DSS-treated) and IL- $10^{-/-}$mice and compared with vehicle-treated and WT mice, respectively. DSS-treated mice $(2.5 \% \mathrm{w} / \mathrm{v}$ for 7 days $)$ had significantly 

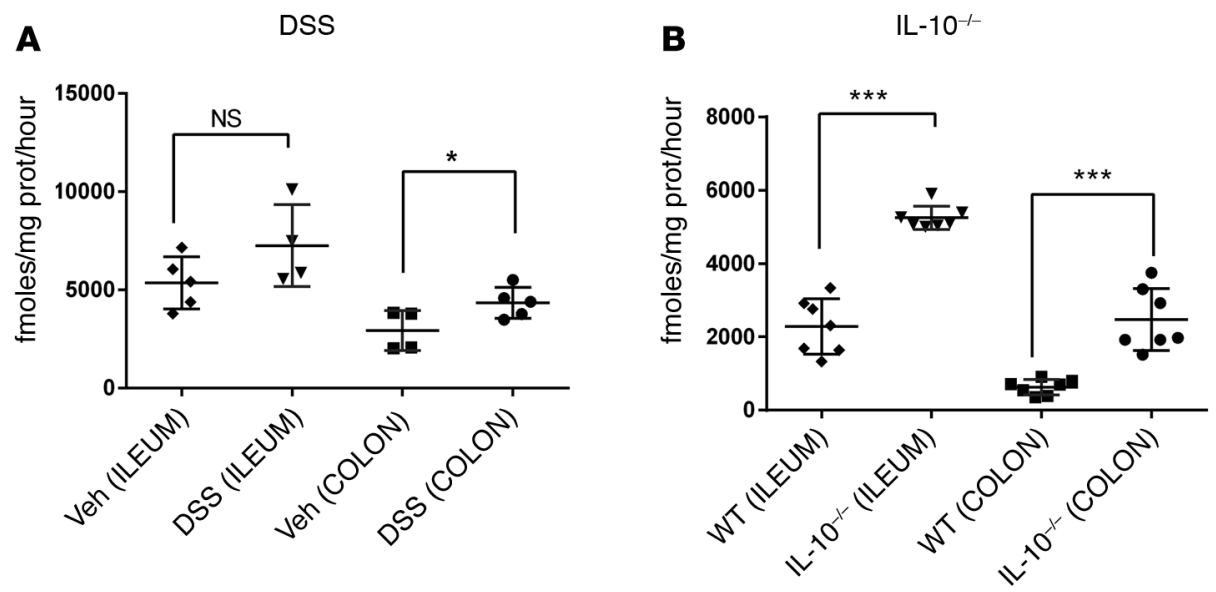

Figure 2. Elevation of FOLH1/GCPII activity in murine models of colitis. FOLH1/glutamate carboxypeptidase II (FOLH1/ GCPII) enzymatic activity was evaluated in colon and ileum samples from two well-validated preclinical models of IBD. (A) Dextran sodium sulfate-treated (DSS-treated) mice showed significant enhancement of FOLH1/GCPII activity versus vehicle-treated mice in colon; a similar trend was observed in the ileum samples, although statistical significance was not achieved $\left({ }^{*} P<0.05\right.$, 2-tailed $t$ test). (B) IL-10 ${ }^{-/-}$mice showed a statistically significant enhancement of FOLH1/ GCPII activity in both inflamed colon and ileum samples versus WT mice. Data are shown as mean \pm SEM. Mouse samples ( $n=4-7$ for each group) were analyzed and assays were performed in triplicate ( ${ }^{* *} P<0.0001,2$-tailed $t$ test).

enhanced enzymatic activity versus vehicle-treated mice in the colon $(1.5 \pm 0.3$-fold; $\mathrm{P}<0.05$; Figure $2 \mathrm{~A})$, with a trend towards an increase in the ileum (1.4 \pm 0.2-fold; NS; Figure 2A). Similarly, FOLH1/GCPII enzymatic activity was also significantly elevated in the inflamed colonic mucosa of 12 - to 16-week-old IL-10-- mice when compared with similarly aged WT mice $(3.9 \pm 0.7$-fold versus WT; $P<0.0001$; Figure 2B). Ileal tissues of IL-10-/ mice also displayed significant enzymatic activity increases ( $2.30 \pm 0.29$-fold versus WT; $P<0.0001$; Figure 2B).

FOLH1/GCPII inhibitor 2-PMPA ameliorates disease activity in the DSS model of colitis. Daily 2-PMPA (100 $\mathrm{mg} / \mathrm{kg}$ i.p.) administration in DSS-treated mice resulted in significantly decreased disease activity index (DAI) scores following 5 days of treatment, based on improved body weight, better stool consistency, and reduced rectal bleeding $(P<0.01$; Figure 3A). To confirm FOLH1/GCPII target engagement, we evaluated both the 2-PMPA drug levels and FOLH1/GCPII inhibitory activity in intestinal samples from DSS-treated mice. FOLH1/GCPII enzymatic activity was $>90 \%$ reduced in 2-PMPA-treated mice, indicating clear target engagement $(P<0.001$; Figure 3B). 2-PMPA drug levels were $23.0 \pm 1.4 \mathrm{nmol} / \mathrm{ml}$ in plasma and $21.4 \pm 1.2 \mathrm{nmol} / \mathrm{g}$ in the colonic mucosa at 2 hours following $100 \mathrm{mg} / \mathrm{kg}$ i.p. dosing, exceeding the drug's $\mathrm{IC}_{50}$ for FOLH1/GCPII (23). These data provide strong support for FOLH1/GCPII target engagement and functional inhibition during the efficacy experiments. Histologically, vehicle-treated mice exhibited more severe mucosal damage, including diminished goblet cells, loss or distorted crypts, neutrophil infiltration, and marked thickening of the muscularis mucosa, submucosa, and muscularis propria, when compared with 2-PMPA-treated mice (Figure 3C).

FOLH1 $1^{-1-}$ mice are resistant to DSS-induced colitis. $\mathrm{FOLH1}^{-1-}$ mice treated with DSS consistently exhibited a lower mean DAI score than their age-matched WT controls $(P<0.05$; Figure $4 \mathrm{~A})$. Mean colon length was also significantly longer in $\mathrm{FOLH1}^{-/-}$mice versus WT controls $(P<0.01$; Figure $4 \mathrm{~B})$, suggesting less overall inflammation. Histologically, while WT mice exhibited massive neutrophil infiltration and loss of crypts and goblet cells, $\mathrm{FOLH1}^{-/-}$mice showed healthier colonic mucosa, with noticeably less neutrophil infiltration and better-preserved crypts and goblet cells (Figure 4C).

FOLH1/GCPII inhibitor 2-PMPA ameliorates spontaneous colitis in the IL-10-1- model. As described previously (24), IL-10-/- mice spontaneously develop colitis that is characterized by a hypertrophic colon and development of rectal prolapse starting at approximately 3 to 4 months of age. In this study, daily treatment with 2-PMPA ( $100 \mathrm{mg} / \mathrm{kg}$ i.p.) was initiated in 16- to 18 -week-old $\mathrm{IL}-10^{-/-}$mice and continued daily for 3 weeks. No change in overall body weight was observed between the groups. However, at sacrifice 2-PMPA-treated mice exhibited significantly less colonic hypertrophy as indicated by a significant decrease in the colon weight $(0.49 \pm 0.06$ g vs. $0.64 \pm 0.07$ g; $P<0.001$; Figure $5 \mathrm{~B})$, better stool consistency (Figure $5 \mathrm{~A})$, and, 
A

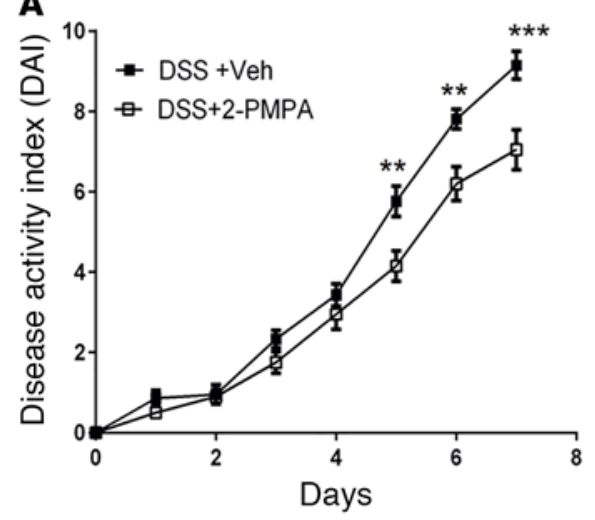

C

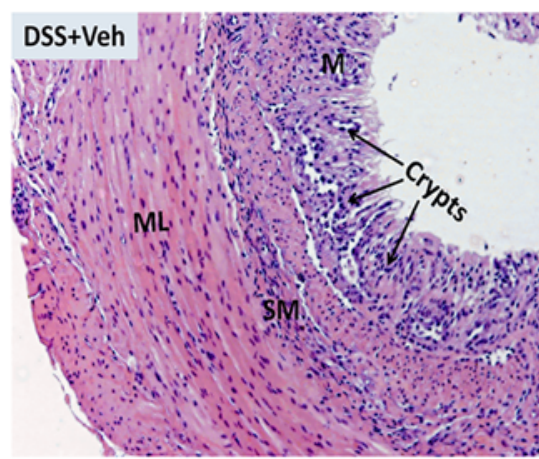

B
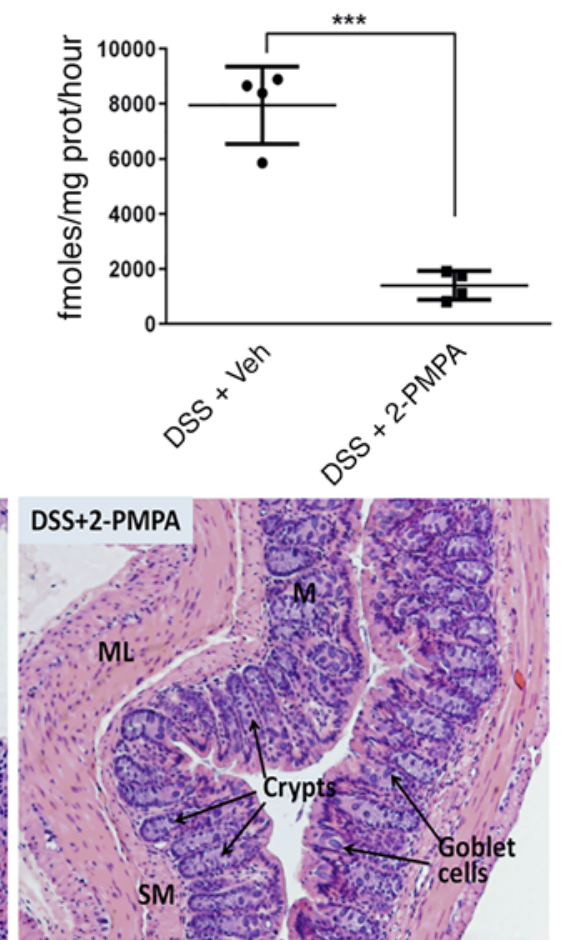

Figure 3. 2-PMPA ameliorates dextran sodium sulfate-induced colitis. (A) Daily 2-PMPA treatment (100 mg/kg i.p.) reduced colitis severity as indicated by the reduced disease activity index (DAI) scores which is a composite score of body weight, stool consistency, diarrhea and intestinal/rectal bleeding. Data are shown as mean \pm SEM ( $n=20$ mice per group) ( ${ }^{* *} P<0.01,{ }^{* *} P<0.001$, 2-tailed $t$ test). (B) 2-PMPA inhibits FOLH1/GCPII enzymatic activity in colonic mucosal extracts from dextran sodium sulfate-treated (DSS-treated) mice by $>90 \%$ confirming target engagement. Mouse samples $(n=4)$ were analyzed per group and assays were performed in triplicate. ( ${ }^{* *} P<0.001,2$-tailed $t$ test). (C) Representative images of H\&E-stained colon sections of 2-PMPA- and vehicle-treated mice with DSS-induced colitis ( $n=5$ per group). Untreated mice exhibited severe colitis, with disrupted epithelial linings, loss of crypts, thickening of the bowel wall, and massive infiltration of the inflammatory cells. 2-PMPA treatment led to protection of the crypts and drastic reduction of the inflammatory cell infiltration. Original magnification, $\times 100$. M, mucosal layer; SM, submucosal; ML, muscular layer

in some cases ( 2 of 10), even prolapse retraction (Supplemental Figure 1; supplemental material available online with this article; doi:10.1172/jci.insight.88634DS1). H\&E staining from the intestines demonstrated remarkably improved histology, including less hypertrophic mucosa, fewer infiltrating neutrophils, and preserved crypts and goblet cells (Figure 5C).

\section{Discussion}

The experiments reported here demonstrate, for the first time, the potential therapeutic effects of FOLH1/ GCPII inhibition in IBD. Increased FOLH1 gene expression was recently reported in biopsy tissue extracted from IBD patients, specifically ileal CD samples $(6,8)$. In fact, the gene was described as a "hub" gene, with significant correlations to 12 of 16 other IBD gene biomarkers (6). These correlations suggested that the gene product of FOLH1, a type II transmembrane glycoprotein termed GCPII (EC 3.4.17.21), might serve not only as an IBD biomarker, but also as a therapeutic target.

We found that relative to non-IBD subjects, the FOLH1/GCPII enzymatic activity was robustly and significantly increased in diseased ileum and colon from both UC and CD subjects. In agreement with the FOLH1 gene expression profile, the largest mean increase (8.6-fold) was seen in ileal CD patients. In addition, the enzymatic activity clearly differentiated diseased versus uninvolved areas within each patient, with increases ranging from 2.8 to 41 -fold. These results demonstrate that the previously reported increase in FOLH1 gene expression (6-8) is associated with increased functional enzymatic activity and is specific for areas affected by disease (see Figure 1). Given that FOLH1/GCPII activity assays are well 
A
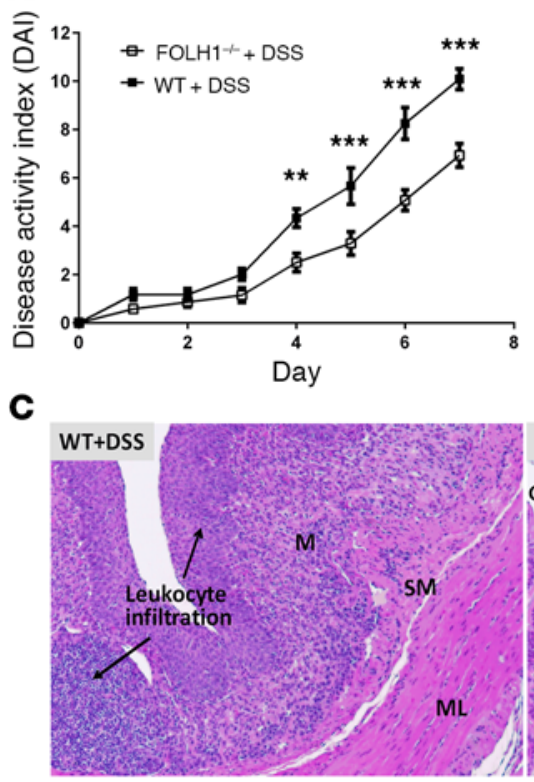

B

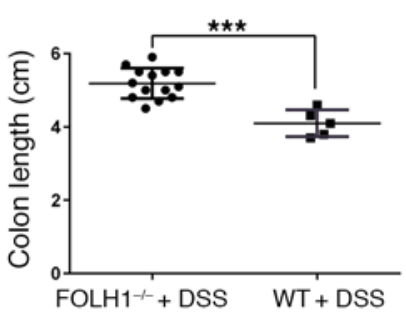

Figure 4. $\mathrm{FOLH1}^{-1-}$ mice are protected against dextran sodium sulfate-induced colitis. $\mathrm{FOLHT}^{-/-}$and WT mice were given dextran sodium sulfate (DSS) in their drinking water for 7 days, and disease activity index (DAI) was monitored daily. Data are shown as mean \pm SEM ( $n=14$ mice/group). (A) $F O L \mathrm{LT}^{-1-}$ mice showed significantly reduced DAl compared with WT mice ( ${ }^{* *} P<0.01$, ${ }^{* *} P<0.001,2$-way ANOVA). (B) $F O L H 1^{-1-}$ mice showed significantly longer colons compared with WT, suggesting reduced inflammation ( $n=5-14$ mice per group) ${ }^{* * *} P<0.001,2$-tailed $t$ test). (C) Histological evaluation confirmed that $\mathrm{FOLH1}^{-1-}$ mice exhibited markedly reduced disease in response to DSS ( $n=5$ per group). WT mice exhibited thickening of the colon wall, including mucosa and muscular layers, as well as massive leukocyte infiltration, loss of crypts, and diminishing goblet cells, while the $\mathrm{FOLH}^{-1-}$ mice showed relatively minor changes, with clearly defined crypts and visible goblet cells, as well as drastically reduced number of inflammatory cell infiltration. Original magnification, $\times 100$. M, mucosal layer; SM, submucosal; ML, muscular layer.

validated and simple to conduct (25), they could be developed as an alternative to current histological methods for IBD diagnosis.

Given the profoundly increased enzymatic activity within diseased areas in patients, we next determined if a similar increase in enzymatic activity occurred in preclinical IBD models. Using the widely employed DSS and IL-10-/- murine models of colitis, we showed a significant increase in FOLH1/GCPII activity in the colons of diseased versus vehicle-treated or WT mice, respectively. A similar significant increase in enzymatic activity was observed in the ileum samples of $\mathrm{IL}-10^{-/-}$mice versus WT mice, while, in DSS mice, an increasing trend versus vehicle-treated mice was observed, but it did not reach significance. The recapitulation of the increased enzymatic activity in a mouse model prompted us to evaluate the therapeutic effect of enzyme inhibition in the mouse IBD model. Systemic administration of the potent and selective FOLH1/GCPII inhibitor, 2-PMPA $(23,26)$, was found to dramatically ameliorate IBD symptoms in the IL $10^{-/-}$model, a finding which was reproduced in DSS-induced colitis. Specifically, 2-PMPA significantly decreased disease severity as measured by disease activity index, colon length, colon weight, stool consistency, and/or histopathology. The drug effect was particularly remarkable in the IL10-/- mice, in which 2-PMPA treatment resulted in a retraction of prolapse in 2 of the 10 mice exhibiting prolapse prior to treatment, a phenomenon that we have not previously observed in our laboratory. This improvement was unequivocal in that the body weight of these prolapse-retracting mice also increased dramatically while the colon weight markedly decreased (healthier colon) (Supplemental Figure 1). We subsequently confirmed that these beneficial effects were due to FOLH1/GCPII inhibition. First, we demonstrated that following systemic administration, the 2-PMPA concentration in the colonic mucosa exceeded the drug's $\mathrm{IC}_{50}$ and resulted in a $>90 \%$ decrease in FOLH1/GCPII enzymatic activity. In addition to the pharmacokinetic/ pharmacologic evidence of target engagement, the role of GCPII was further confirmed in $\mathrm{FOLH1}^{-/-}$mice, as they were found to be significantly protected from DSS-induced colitis when compared with WT mice. 


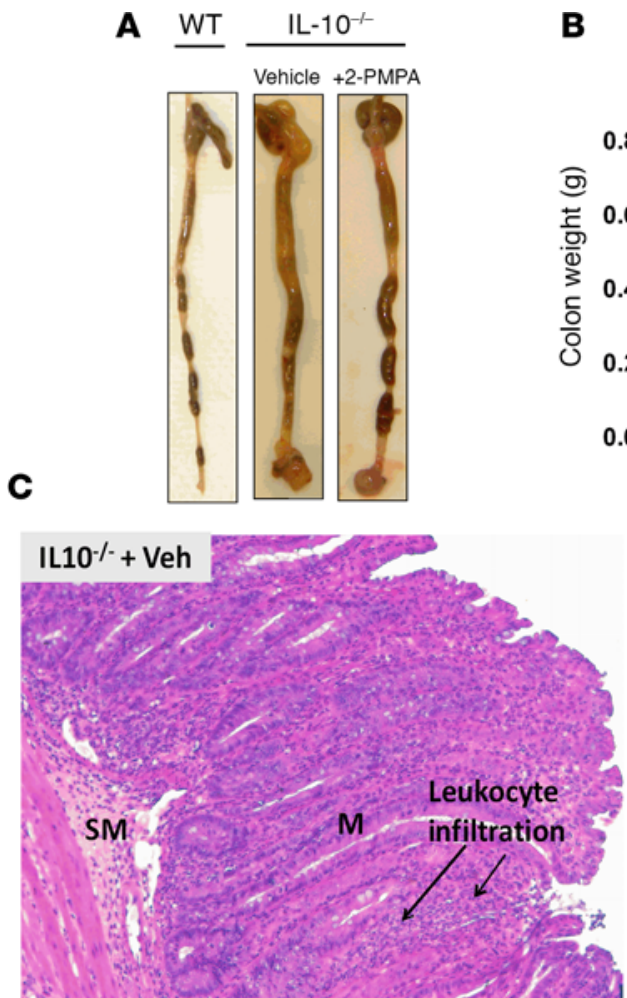

B
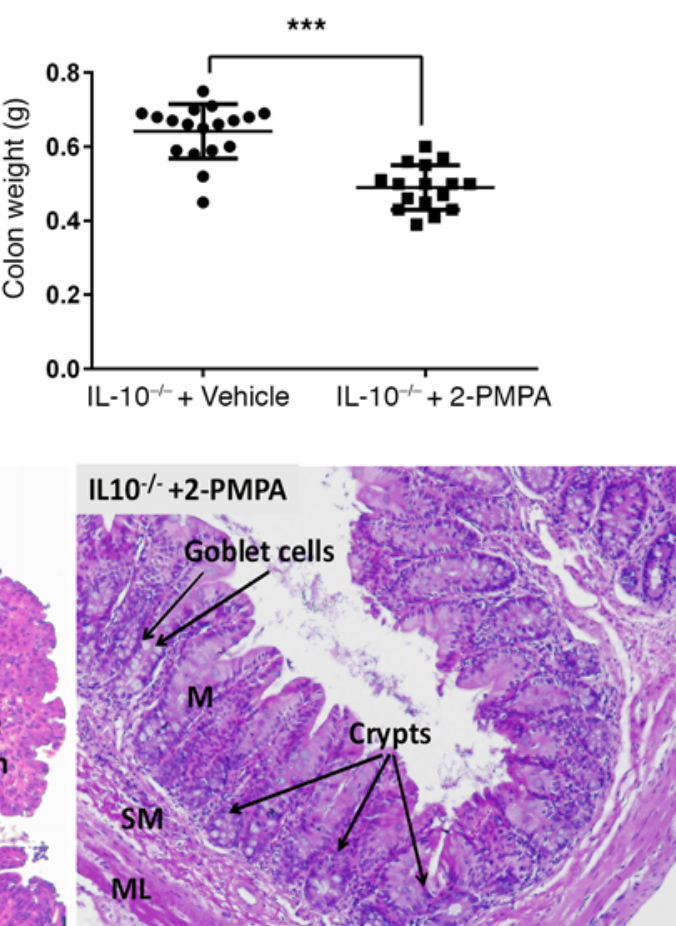

Figure 5. 2-PMPA ameliorates disease activity in a IL-10-/- model of spontaneous colitis. IL-10-/- mice (16-18 weeks old) were treated with daily 2 -PMPA (100 mg/kg i.p.) for 3 weeks. Data are shown as mean \pm SEM ( $n=18-20$ mice per group). (A) 2-PMPA provided better stool consistency. (B) 2-PMPA improved colon weight ${ }^{* * *} P<0.001$, 2-tailed $t$ test) (C) Histological evaluation showed that 2-PMPA treatment led to a healthier colon. Untreated mice exhibited marked thickening of the colon wall as a result of excessive hyperplasia, massive leukocyte infiltration, loss of crypts, and diminishing of goblet cells, while the 2-PMPA-treated group showed relatively minor changes. Original magnification, $\times 100$. M, mucosal layer; SM, submucosal; ML, muscular layer.

While the efficacy of FOLH1/GCPII inhibition in IBD preclinical models is clear, the site of action and therapeutic mechanism has yet to be determined. With regard to site of action, although the increased expression in IBD patients has been reported in epithelial cells (6) it is also possible that inhibition of FOLH1/ GCPII may affect the enteric nervous system or infiltrating immune cells. Of interest, our laboratory recently found that GCPII is highly upregulated in microglial cells (e.g., the immune cells of the brain) following inflammation and its inhibition prevents neurological damage (27). With respect to enzymatic mechanism, to date there are 3 known FOLH1/GCPII substrates that could be implicated as possible mediators of the therapeutic effect, including N-acetylaspartylglutamate (NAAG) (28), folate polyglutamate (29-31), and laminin-derived peptides $(32,33)$. NAAG is one of the most abundant peptides in nervous tissues where it is thought to participate in glutamatergic transmission, acting through NMDA and mGlu3 receptors (20, 34, 35). Although little is known about NAAG in the enteric nervous system, its receptors have been reported in the gut (36). Similar to FOLH1/GCPII upregulation in IBD, increased enzyme activity has been reported in a variety of nervous system diseases (36-42), including neuroinflammation (36, 41-44). In order to determine the role of NAAG and its receptors in IBD, more detailed studies on NAAG localization, synthesis, and regulation under both normal conditions and in IBD are required. It would also be of interest to examine the effects of agonists and antagonists of these receptors on disease. A second known substrate is folate poly- $\gamma$-glutamate. The enzyme is responsible for sequential removal of the multiple terminal $\gamma$-linked glutamates to generate folic acid, which is actively absorbed by the reduced folate carrier. It is possible that elevated folate hydrolase activity in IBD provides improved folate availability from the gut to support nucleotide biosynthesis and cell division of inflammatory cells. In support of this possibility, antifolate drugs such as methotrexate are an effective IBD treatment (45). Finally, there is a possible antiangiogenic effect of FOLH1/ GCPII inhibition in IBD. The enzyme has been hypothesized to be a proangiogenic surface protease, which 
is highly upregulated in the neovasculature of solid tumors $(10,33,46,47)$. FOLH1/GCPII is thought to hydrolyze laminin peptides to liberate fragments that enhance endothelial cell adhesion and migration (32). Importantly, enhanced angiogenesis has been a characteristic feature of both CD and UC (48, 49), and angiogenesis blockade has been shown to effectively ameliorate experimental colitis (50-52). Our laboratory has recently identified a specific angiogenic dipeptide derived from GCPII cleavage of laminin (53) and plans to explore its regulation in IBD with/without GCPII treatment. In summary, we have used a human-to-mouse strategy and translated an unexpected finding in a human IBD GWAS study to identify a therapeutic approach. Specifically, we have demonstrated a large and significant increase of glutamate carboxypeptidase activity in clinical biopsy samples from disease-affected areas compared with unaffected areas in both CD and UC. We then moved to animal models showing a similar enzymatic increase associated with disease. Subsequently, we showed that systemic administration of a potent and selective FOLH1/ GCPII inhibitor could ameliorate IBD symptoms and that $\mathrm{FOLH}^{-1-}$ mice are resistant to disease. We are currently working to identify the relevant substrates and receptors affected by FOLH1/GCPII inhibition in the gut, which may lead to additional therapeutic targets. While further mechanistic studies are underway, the present data strongly support the utility of FOLH1/GCPII as both a biomarker and therapeutic target in IBD. Our findings lay the foundation for rapid development and clinical translation of FOLH1/GCPII inhibitors for the treatment of IBD.

\section{Methods}

2-PMPA was synthesized by our laboratory using methods reported previously (23). LC/MS-grade acetonitrile and water with $0.1 \%$ formic acid were obtained from Fisher Scientific. DSS was obtained from Affymetrix, and heparinized rat plasma was obtained from Innovative Research Inc. All other chemical and reagents were purchased from Sigma-Aldrich.

FOLH1/GCPII enzymatic activity in patient samples. Surgically resected intestinal mucosa from patients with CD and UC and non-IBD subjects were donated by the Johns Hopkins Gastroenterology Department (Baltimore, Maryland, USA). Tissue samples (intestinal mucosa), from both involved (diseased) and uninvolved (no disease) colon or ileum of IBD patients or normal intestine of healthy controls, were obtained from surgically resected intestine or endoscopic biopsies and stored immediately in liquid nitrogen. Glutamate carboxypeptidase activity in the samples was measured using a previously described radioenzymatic assay by our laboratory $(54,55)$. In brief, intestinal tissues were weighed and immersed in $0.5 \mathrm{ml}$ of ice-cold $50 \mathrm{mM}$ Tris buffer ( $\mathrm{pH} 7.7$ at room temperature). Each tissue was sonicated for 30 to 60 seconds using an ultrasonic cell disrupter. After a 2 minute spin at 13,000 g, supernatants were analyzed for protein content and glutamate carboxypeptidase activity.

DSS murine model of colitis. Six- to eight-week-old adult male C57BL/6 mice were obtained from Jackson Laboratories. The animals were housed in the Johns Hopkins animal facility under controlled temperature $\left(25^{\circ} \mathrm{C}\right)$ and photoperiods (12-hour-light/12-hour-dark cycle) with access to standard diet and water (or specified DSS-containing drinking water) ad libitum. Mice were housed 5 per cage and allowed to acclimate to these conditions for at least 7 to 10 days before inclusion in experiments.

For the functional enzymatic activity assessment, mice were randomized before the start of the DSS treatment on the basis of body weight and age into two equivalent experimental groups ( $n=5 /$ group), including vehicle and DSS plus vehicle. Acute colitis was induced by administration of $2.5 \%(\mathrm{w} / \mathrm{v})$ DSS (MW 40,000-50,000) in the drinking water for 7 days as described previously $(56,57)$. On day 8 , the colon and ileum were removed and feces were flushed clean with cold saline using a syringe and weighed. Glutamate carboxypeptidase activity was then measured in the samples using our previously described radioenzymatic assay $(54,55)$, which is detailed above.

For the efficacy studies, mice ( $n=20$ per group) were similarly randomized before the start of the DSS treatment on the basis of body weight and age into three equivalent experimental groups: (a) vehicle, (b) DSS plus vehicle, and (c) DSS plus 2-PMPA. Acute colitis was induced by administration of 2.5\% (w/v) DSS (MW 40,000-50,000) in the drinking water for 7 days. Daily dosing of i.p. vehicle (HBSS) or 2-PMPA $(100 \mathrm{mg} / \mathrm{kg})$ began when DSS was initiated and was continued until sacrifice on day 8 . Disease status was monitored using a DAI, including body weight, stool consistency, diarrhea, and intestinal/rectal bleeding, based on well-established scores that simulate clinical IBD presentations (58). Specifically, the following criteria were used: (a) weight loss $(0=$ none; $1=1 \%-5 \%$ loss; $2=5 \%-10 \%$ loss; $3=10 \%-15 \%$ loss; $4=$ more than $15 \%$ loss); (b) stool consistency ( $0=$ normal; $1=$ slightly soft; $2=$ soft but still formed; $3=$ soft 
and not formed; 4 = watery diarrhea); and (c) bleeding ( 0 = negative; $1=$ blood test $+; 2=$ blood test ++ ; $3=$ visible blood trace in stool; $4=$ rectal bleeding), as assessed by the ColoScreen occult blood test (Helena Laboratories). For the knockout mouse studies, $\mathrm{FOLH1}^{-/-}$mice were provided by Joseph Neale (Georgetown University, Washington, DC, USA), who had received them originally from Warren Heston (Cleveland Clinic Lerner Research Institute, Cleveland, Ohio, USA) (59). The mice had been backcrossed at least 10 times to C57BL/6. For this study, male FOLH1 $1^{-1}$ mice and C57BL/6J WT mice (6-8 weeks old) were given $2.5 \%$ DSS w/v in their drinking water for 7 days, and their DAI was monitored as detailed above.

IL-10-1- murine model of spontaneous colitis. C57BL/6 IL-10-/-, 6- to 8-week-old, 18-22 g mice were obtained from Jackson Laboratories. Animals were housed under specific pathogen-free conditions at the Johns Hopkins animal facility for approximately 10 weeks before the beginning of the experiment to allow spontaneous disease onset.

For the functional enzymatic assay assessments, 12- to 16-week-old IL-10-1- and age-matched WT mice were used. Colon and ileum were removed, feces were flushed, intestinal samples were weighed, and glutamate carboxypeptidase activity in the samples was measured using a radioenzymatic assay previously described by our laboratory $(54,55)$ and detailed above.

For the efficacy studies, 16- to 18-week-old IL-10 $0^{-/-}$mice were divided to 2 groups (with $18-20$ mice per group) and given daily i.p. injection of vehicle (HBSS) or $100 \mathrm{mg} / \mathrm{kg}$ 2-PMPA for 3 weeks before sacrifice. Body weight and rectal prolapse were measured and recorded daily. Following sacrifice, the abdomen of the mice was opened by a ventral midline incision. The colon was removed and feces were flushed clean with cold saline using a syringe. Colon weight, length, and stool consistency were then determined as we have previously described (57).

Histological evaluation. Five-mm segments of proximal and distal colon were resected and fixed in $10 \%$ neutral buffered formalin for histological examination as described previously (57). Briefly, tissues were embedded in paraffin wax, sectioned into $4-\mu \mathrm{m}$-thick sections with a paraffin microtome (ThermoFisher), and mounted on microscope slides (Fisher Scientific). Dewaxed sections were stained with H\&E (Richard Alen Scientific). Histological images were obtained using a Zeiss Axio Observer microscope with an Olympus DP72 camera (Zeiss).

FOLH1 target engagement studies. To confirm that 2-PMPA was engaging and inhibiting the FOLH1/ GCPII enzyme, glutamate carboxypeptidase enzymatic activity measurements in colonic mucosa were assessed in mice at the end of the efficacy studies at 2 hour following the last 2-PMPA dose. Tissues were weighed and immersed in $0.5 \mathrm{ml}$ of ice-cold $50 \mathrm{mM}$ Tris buffer ( $\mathrm{pH} 7.7$ at room temperature). While on ice, each tissue was sonicated for 30 to 60 seconds (medium output, 60) using a Kontes ultrasonic cell disrupter. After a 2 minute spin at $13,000 \mathrm{~g}$, supernatants were analyzed for protein content (DC Protein Assay Kit; Bio-Rad) and glutamate carboxypeptidase activity as per our published procedures $(54,55)$.

2-PMPA analysis in plasma and colon. Two hours following the last 2-PMPA dose in the DSS efficacy studies, mice were anesthetized in a chamber containing isoflurane and then sacrificed by cervical dislocation, and blood and colonic mucosa were collected for 2-PMPA bioanalysis. Plasma was generated from blood by centrifugation, and samples were stored at $-80^{\circ} \mathrm{C}$ until bioanalysis. Concentrations of 2-PMPA in plasma and colon mucosa were determined via LC/MS/MS, as we have previously described (60). Briefly, 2-PMPA was extracted from plasma and tissue by protein precipitation with $6 \mathrm{X}$ methanol containing 2-(phosphonomethyl) succinic acid (2-PMSA; $1 \mu \mathrm{M})$ as an internal standard. The samples were vortexed (30 s) and centrifuged (10,000 g for $10 \mathrm{~min})$. Supernatants were dried under a gentle stream of nitrogen at $45^{\circ} \mathrm{C}$ and the residue reconstituted with $100 \mu \mathrm{l}$ of acetonitrile. $50 \mu \mathrm{l}$ of the derivatizing agent $\mathrm{N}$-tert-Butyldimethysilyl-N-methyltrifluoro-acetamide (MTBSTFA) was added, and samples were heated at approximately $60^{\circ} \mathrm{C}$ for 40 minutes. At the end of 40 minutes, the derivatized samples were analyzed via LC/MS/MS. Chromatographic analysis was performed using an Accela ultra high-performance system consisting of an analytical pump and an autosampler coupled with TSQ Vantage mass spectrometer (Thermo Fisher Scientific Inc.).

Statistics. All data, except for that shown in Figure 1B, is presented as mean \pm SEM. Statistical analyses were performed using Graph Pad Prism 6.0. Comparisons between groups in human and animal studies were made with 2-tailed $t$ tests. Evaluation of differences in disease activity indices was performed with 2-way ANOVA. $P$ values of less than 0.05 were considered statistically significant for all data.

Study approval. Surgically resected intestinal mucosa from patients with CD and UC and non-IBD subjects were donated by the Johns Hopkins Gastroenterology Department. Specimen collection was 
approved by the Johns Hopkins University Review Board (NA00038329/CR00009103), and the diagnosis was based on the pathology report issued by a well-trained pathologist assigned to each respective case. All patients signed a written consent form to participate. The experimental protocol (MO16M13) for murine IBD studies was approved by the Institutional Animal Care and Use Committee of Johns Hopkins University and adhered to all of the applicable institutional and governmental guidelines for the humane treatment of laboratory animals.

\section{Author contributions}

BSS, XL, RR, and WJ participated in research design. RR, WJ, HZ, KRH, AGT, KMW, and MS conducted experiments. XL, BSS, MM, and CR contributed reagents or analytic tools. RR, KMW, WJ, HZ, AGT, and MS performed data analysis. RR, BSS, JJV, and XL contributed to the writing of the manuscript.

\section{Acknowledgments}

This project was supported by NIH grant RO1CA161056 (to BSS), NIH grant P30DK089502 (to XL), and TEDCO Maryland Innovation Initiative award (to BSS and RR).

Address correspondence to: Barbara S. Slusher, Johns Hopkins Drug Discovery, Johns Hopkins School of Medicine, 855 North Wolfe Street, Baltimore, Maryland 21205, USA. Phone: 410.614 .0662 or 410.960.6162; E-mail: bslusher@jhmi.edu. Or to: Xuhang Li, Division of Gastroenterology, Department of Medicine, Johns Hopkins University School of Medicine, 720 Rutland Avenue, Ross 746, Baltimore, Maryland 21205, USA. Phone: 443.502.4487; E-mail: xuhang@jhmi.edu.

1. Xavier RJ, Podolsky DK. Unravelling the pathogenesis of inflammatory bowel disease. Nature. 2007;448(7152):427-434

2. Strober W, Fuss I, Mannon P. The fundamental basis of inflammatory bowel disease. J Clin Invest. 2007;117(3):514-521

3. Sartor RB. Mechanisms of disease: pathogenesis of Crohn's disease and ulcerative colitis. Nat Clin Pract Gastroenterol Hepatol. 2006;3(7):390-407.

4. Kaser A, Zeissig S, Blumberg RS. Inflammatory bowel disease. Annu Rev Immunol. 2010;28:573-621.

5. Hamilton MJ, Snapper SB, Blumberg RS. Update on biologic pathways in inflammatory bowel disease and their therapeutic relevance. J Gastroenterol. 2012;47(1):1-8.

6. Zhang T, et al. An ileal Crohn's disease gene signature based on whole human genome expression profiles of disease unaffected ileal mucosal biopsies. PLoS One. 2012;7(5):e37139.

7. Ben-Shachar S, et al. Gene expression profiles of ileal inflammatory bowel disease correlate with disease phenotype and advance understanding of its immunopathogenesis. Inflamm Bowel Dis. 2013;19(12):2509-2521.

8. Noble CL, et al. Characterization of intestinal gene expression profiles in Crohn's disease by genome-wide microarray analysis. Inflamm Bowel Dis. 2010;16(10):1717-1728.

9. Chang SS. Overview of prostate-specific membrane antigen. Rev Urol. 2004;6 Supp1 10:S13-S18.

10. Chang SS, O'Keefe DS, Bacich DJ, Reuter VE, Heston WD, Gaudin PB. Prostate-specific membrane antigen is produced in tumor-associated neovasculature. Clin Cancer Res. 1999;5(10):2674-2681.

11. Grant CL, et al. Prostate specific membrane antigen (PSMA) regulates angiogenesis independently of VEGF during ocular neovascularization. PLoS One. 2012;7(7):e41285.

12. Slusher BS, Tsai G, Yoo G, Coyle JT. Immunocytochemical localization of the N-acetyl-aspartyl-glutamate (NAAG) hydrolyzing enzyme N-acetylated alpha-linked acidic dipeptidase (NAALADase). J Comp Neurol. 1992;315(2):217-229.

13. Bacich DJ, Pinto JT, Tong WP, Heston WD. Cloning, expression, genomic localization, and enzymatic activities of the mouse homolog of prostate-specific membrane antigen/NAALADase/folate hydrolase. Mamm Genome. 2001;12(2):117-123

14. Ferraris DV, Shukla K, Tsukamoto T. Structure-activity relationships of glutamate carboxypeptidase II (GCPII) inhibitors. Curr Med Chem. 2012;19(9):1282-1294.

15. Wang H, et al. Bioisosterism of urea-based GCPII inhibitors: Synthesis and structure-activity relationship studies. Bioorg Med Chem Lett. 2010;20(1):392-397.

16. Majer P, et al. Discovery of orally available prodrugs of the glutamate carboxypeptidase II (GCPII) inhibitor 2-phosphonomethylpentanedioic acid (2-PMPA). J Med Chem. 2016;59(6):2810-2819.

17. Novakova Z, et al. Unprecedented binding mode of hydroxamate-based inhibitors of glutamate carboxypeptidase II: structural characterization and biological activity. JMed Chem. 2016;59(10):4539-4550

18. Vornov JJ, et al. Still NAAG'ing after all these years: the continuing pursuit of GCPII inhibitors. Adv Pharmacol. 2016;76:215-255.

19. Bařinka C, Rojas C, Slusher B, Pomper M. Glutamate carboxypeptidase II in diagnosis and treatment of neurologic disorders and prostate cancer. Curr Med Chem. 2012;19(6):856-870.

20. Slusher BS, et al. Selective inhibition of NAALADase, which converts NAAG to glutamate, reduces ischemic brain injury. Nat Med. 1999;5(12):1396-1402.

21. Zhou J, Neale JH, Pomper MG, Kozikowski AP. NAAG peptidase inhibitors and their potential for diagnosis and therapy. Nat Rev Drug Discov. 2005;4(12):1015-1026.

22. Neale JH, Olszewski RT, Gehl LM, Wroblewska B, Bzdega T. The neurotransmitter N-acetylaspartylglutamate in models of pain, ALS, diabetic neuropathy, CNS injury and schizophrenia. Trends Pharmacol Sci. 2005;26(9):477-484. 
23. Jackson PF, et al. Design, synthesis, and biological activity of a potent inhibitor of the neuropeptidase N-acetylated alpha-linked acidic dipeptidase. J Med Chem. 1996;39(2):619-622.

24. Kühn R, Löhler J, Rennick D, Rajewsky K, Müller W. Interleukin-10-deficient mice develop chronic enterocolitis. Cell. 1993;75(2):263-274.

25. Rojas C, et al. Glutamate carboxypeptidase activity in human skin biopsies as a pharmacodynamic marker for clinical studies. $J$ Transl Med. 2011;9:27.

26. Jackson PF, et al. Design and pharmacological activity of phosphinic acid based NAALADase inhibitors. $J$ Med Chem. 2001;44(24):4170-4175

27. Zhang Z, et al. Maternal inflammation leads to impaired glutamate homeostasis and up-regulation of glutamate carboxypeptidase II in activated microglia in the fetal/newborn rabbit brain. Neurobiol Dis. 2016;94:116-128.

28. Vornov JJ, et al. Still NAAG'ing after all these years: the continuing pursuit of GCPII inhibitors. Adv Pharmacol. 2016;76:215255

29. Cheng TY, et al. Folate-mediated one-carbon metabolism genes and interactions with nutritional factors on colorectal cancer risk: Women's Health Initiative Observational Study. Cancer. 2015;121(20):3684-3691.

30. Guo J, et al. The maternal folate hydrolase gene polymorphism is associated with neural tube defects in a high-risk Chinese population. Genes Nutr. 2013;8(2):191-197.

31. Navrátil M, et al. Structural and biochemical characterization of the folyl-poly- $\gamma$-1-glutamate hydrolyzing activity of human glutamate carboxypeptidase II. FEBS J. 2014;281(14):3228-3242.

32. Conway RE, et al. Prostate specific membrane antigen produces pro-angiogenic laminin peptides downstream of matrix metalloprotease-2. Angiogenesis. 2013;16(4):847-860.

33. Conway RE, Petrovic N, Li Z, Heston W, Wu D, Shapiro LH. Prostate-specific membrane antigen regulates angiogenesis by modulating integrin signal transduction. Mol Cell Biol. 2006;26(14):5310-5324.

34. Khacho P, Wang B, Ahlskog N, Hristova E, Bergeron R. Differential effects of N-acetyl-aspartyl-glutamate on synaptic and extrasynaptic NMDA receptors are subunit- and $\mathrm{pH}$-dependent in the CA1 region of the mouse hippocampus. Neurobiol Dis 2015;82:580-592.

35. Urazaev AK, Grossfeld RM, Lieberman EM. Regulation of glutamate carboxypeptidase II hydrolysis of N-acetylaspartylglutamate (NAAG) in crayfish nervous tissue is mediated by glial glutamate and acetylcholine receptors. JNeurochem. 2005;93(3):605-610

36. Julio-Pieper M, Flor PJ, Dinan TG, Cryan JF. Exciting times beyond the brain: metabotropic glutamate receptors in peripheral and non-neural tissues. Pharmacol Rev. 2011;63(1):35-58.

37. Meyerhoff JL, Carter RE, Yourick DL, Slusher BS, Coyle JT. Genetically epilepsy-prone rats have increased brain regional activity of an enzyme which liberates glutamate from N-acetyl-aspartyl-glutamate. Brain Res. 1992;593(1):140-143.

38. Tsai G, et al. Abnormal excitatory neurotransmitter metabolism in schizophrenic brains. Arch Gen Psychiatry. 1995;52(10):829836

39. Guilarte TR, et al. Dysregulation of glutamate carboxypeptidase II in psychiatric disease. Schizophr Res. 2008;99(1-3):324-332.

40. Ghose S, et al. Glutamate carboxypeptidase II gene expression in the human frontal and temporal lobe in schizophrenia. Neuropsychopharmacology. 2004;29(1):117-125

41. Hayashi M, Morimoto R, Yamamoto A, Moriyama Y. Expression and localization of vesicular glutamate transporters in pancreatic islets, upper gastrointestinal tract, and testis. J Histochem Cytochem. 2003;51(10):1375-1390.

42. Carlton SM, Hargett GL, Coggeshall RE. Localization and activation of glutamate receptors in unmyelinated axons of rat glabrous skin. Neurosci Lett. 1995;197(1):25-28.

43. Yamamoto T, Nozaki-Taguchi N, Sakashita Y. Spinal N-acetyl-alpha-linked acidic dipeptidase (NAALADase) inhibition attenuates mechanical allodynia induced by paw carrageenan injection in the rat. Brain Res. 2001;909(1-2):138-144.

44. Miller KE, Hoffman EM, Sutharshan M, Schechter R. Glutamate pharmacology and metabolism in peripheral primary afferents: physiological and pathophysiological mechanisms. Pharmacol Ther. 2011;130(3):283-309.

45. Inoue K, Yuasa H. Molecular basis for pharmacokinetics and pharmacodynamics of methotrexate in rheumatoid arthritis therapy. Drug Metab Pharmacokinet. 2014;29(1):12-19.

46. Horoszewicz JS, Kawinski E, Murphy GP. Monoclonal antibodies to a new antigenic marker in epithelial prostatic cells and serum of prostatic cancer patients. Anticancer Res. 1987;7(5B):927-935.

47. Liu H, et al. Monoclonal antibodies to the extracellular domain of prostate-specific membrane antigen also react with tumor vascular endothelium. Cancer Res. 1997;57(17):3629-3634.

48. Danese S, et al. Angiogenesis as a novel component of inflammatory bowel disease pathogenesis. Gastroenterology. 2006;130(7):2060-2073

49. Knod JL, Crawford K, Dusing M, Collins MH, Chernoguz A, Frischer JS. Angiogenesis and vascular endothelial growth factor-A expression associated with inflammation in pediatric Crohn's disease. J Gastrointest Surg. 2016;20(3):624-630.

50. Danese S, et al. Angiogenesis blockade as a new therapeutic approach to experimental colitis. Gut. 2007;56(6):855-862.

51. Chidlow JH, et al. Differential angiogenic regulation of experimental colitis. Am J Pathol. 2006;169(6):2014-2030

52. Danese S, et al. Critical role of the CD40 CD40-ligand pathway in regulating mucosal inflammation-driven angiogenesis in inflammatory bowel disease. Gut. 2007;56(9):1248-1256.

53. Conway RE, et al. Prostate specific membrane antigen produces pro-angiogenic laminin peptides downstream of matrix metalloprotease-2. Angiogenesis. 2013;16(4):847-860.

54. Rojas C, Frazier ST, Flanary J, Slusher BS. Kinetics and inhibition of glutamate carboxypeptidase II using a microplate assay. Anal Biochem. 2002;310(1):50-54.

55. Robinson MB, Blakely RD, Couto R, Coyle JT. Hydrolysis of the brain dipeptide N-acetyl-L-aspartyl-L-glutamate. Identification and characterization of a novel $\mathrm{N}$-acetylated alpha-linked acidic dipeptidase activity from rat brain. J Biol Chem. 1987;262(30):14498-14506.

56. Alex P, et al. Clcn5 knockout mice exhibit novel immunomodulatory effects and are more susceptible to dextran sulfate sodium-induced colitis. J Immunol. 2010;184(7):3988-3996. 
57. Alex P, et al. Distinct cytokine patterns identified from multiplex profiles of murine DSS and TNBS-induced colitis. Inflamm Bowel Dis. 2009;15(3):341-352.

58. Wirtz S, Neufert C, Weigmann B, Neurath MF. Chemically induced mouse models of intestinal inflammation. Nat Protoc. 2007;2(3):541-546.

59. Bacich DJ, et al. Deletion of the glutamate carboxypeptidase II gene in mice reveals a second enzyme activity that hydrolyzes N-acetylaspartylglutamate. J Neurochem. 2002;83(1):20-29.

60. Rais R, et al. Bioanalytical method for evaluating the pharmacokinetics of the GCP-II inhibitor 2-phosphonomethyl pentanedioic acid (2-PMPA). J Pharm Biomed Anal. 2014;88:162-169. 